\title{
Pregnancy and Pregnancy Tumour - Is there any relation? A Report of two cases and its pathogenesis
}

\author{
Ramesh KSV ${ }^{1 *}$, Swetha $P^{2}$, Sameera ASS ${ }^{3}$ \\ ${ }^{1}$ Ramesh. K.S.V.,Reader, Department of Periodontics and Implantology, Vishnu Dental College, Bhimavaram, Andhra Pradesh. \\ ${ }^{2}$ Swetha P, Reader, Department of Oral \& Maxillofacial Pathology, Vishnu Dental College, Bhimavaram,Andhra Pradesh. \\ ${ }^{3}$ Sameera ASS, Reader, Department of Oral Medicine \& Radiology, CKS Teja Dental College, Tirupathi, Andhra Pradesh

\section{Abstract}

Granuloma pyogenicum is the second most common oral lesion, which does not produce pus. This is a smooth, painless, non neoplastic and lobulated or cauliflower like growth of the connective tissue seen commonly on gingiva and in young females comparing to males. Pyogenic granuloma seems to contribute $19.76-25 \%$ of all reactive lesions. Pyogenic granuloma being benign in nature this may attain an alarming size causing pain and discomfort. Thus early intervention through reinforcement of adequate oral hygiene measures along with surgical excision down to the periosteum is necessary to minimize the recurrence.

Key words:Granuloma pyogenicum, non neoplastic growth, pregnancy, progesterone and estrogen.

\section{INTRODUCTION}

Often soft tissue enlargements of the oral cavity throw a challenge to a dentist in concluding the diagnosis with its diversified etiopathogenesis. Aetiology of these enlargements could be of either inflammatory, developmental etc., among which, commonly seen is inflammatory hyperplastic growth.[1] Though, there is abundant literature available on this lesion, its occurrence during the pregnancy is still unclear. This article emphasizes mainly on the probable causes for its occurrence during pregnancy.

Pyogenic granuloma (PG) is a hyperactive benign inflammatory lesion and the third most common lesion in the oral cavity[2]. The term "pyogenic granuloma" is a misnomer because the lesion neither contains pus nor it is granulomatous.[3]

Zafarzadeh et al defined pyogenic granuloma as an inflammatory overgrowth of the oral mucosa caused by minor trauma or irritation.[4] Pyogenic granuloma is a painless, soft, sessile with deep red to reddish purple in colour. It has a wide array of appearances and has been referred to by a variety of names such as granuloma pediculatumbenignum, benign vascular tumor, pregnancy tumor, vascular epulis, Crocker and Hartzell's disease. Cawson et al named it as 'granuloma telangiectactium' due to its numerous blood vessels [5]. It is a smooth (or) lobulated mass usually pendunculated, although some are sessile. The surface characteristically shows ulcerated bands ranging from pink to red to purple, depending on age of the lesion and young granulomas are highly vascular. Older lesions tend to become more collagenized and pink. Though, the etiology of pyogenic granuloma is unknown, it usually arises in response to various stimuli such as traumatic injury, low-grade irritation, hormonal factors (or) certain kind of drugs (like cortisone, oral contraceptives, diabetic medication or even allogenic bone marrow transplantation). It may exhibit rapid growth which may create alarm for both patient and clinician, who fear that the lesion might be malignant. Usually this occurs as a single or Multiple/generalized lesion. Commonly seen on gingiva, (75\%)

\section{Address for correspondence* \\ Ramesh KSV \\ Reader, \\ Department of Periodontics and Implantology, \\ Vishnu Dental College, Bhimavaram -534202,Andhra Pradesh}

and in females comparing to males especially during pregnancy. Hence, it is also called as "pregnancy tumor" or "pregnancy epulis" where, it is believed that female sex hormones play a major role in the pathogenesis of the same. Extra gingivally, it can occur on the lips, tongue, buccal mucosa, palate, and the like.[1] pyogenic granuloma is also known as Satellitosis when reported on skin. However, there is sparse evidence to support satellitosis in the oral cavity.[6]

\section{CASE REPORT 1}

A female pregnant(2nd trimester) patient of age 19yrs visited to the clinic with a chief complaint of swelling in the lower left posterior region lasting for 2-3months which started as a small lesion and gradually increasing in size. On extra oral examination facial asymmetry was noticed. On intra oral examination lobulated swelling with slough of measuring about $2.5 \mathrm{~cm} \mathrm{X} 3 \mathrm{cms}$ size is seen is relation to 46 and 47 ( fig 1 ) with a pedunculated base extending to lingual surface of 46 and 47 with established signs of inflammation. On palpation swelling had a rough texture which is firm in consistency with spontaneous bleeding on probing. Lesion was painless and asymptomatic and patient complains of discomfort while chewing and closing the mouth. Interestingly, there were no signs of local etiological factors and mobility of teeth pertaining to the lesion site. With the above factors a provisional diagnosis was made as pyogenic granuloma.

Before proceeding for the surgery routine blood investigations reveled normal values. Complete oral prophylaxis was done. By considering the patient systemic status (i.e. pregnancy) patient was asked to rinse her mouth with diluted betadine and surgery was performed by using electrocautery (fig 2).The lesion was excised from its base including part of normal tissue by using electrosurgical wire tip and confirmed that there were no remnants of the lesion were left to prevent its recurrence (fig 3). After the excision periodontal pack (coepak) was given (fig 4). Antibiotics and analegesics were prescribed and the excised tissue (fig 5) was sent for hispathological examination (fig 6). Patient was recalled for the reassessment after 3 months and found that there was no recurrence.

\section{CASE REPORT 2}

A female pregnant( $2 \mathrm{nd}$ trimester) patient of age $22 \mathrm{yrs} \mathrm{had}$ visited to the dental clinic with a complaint of swelling in the lower left posterior region lasting 1 month which started as a small 


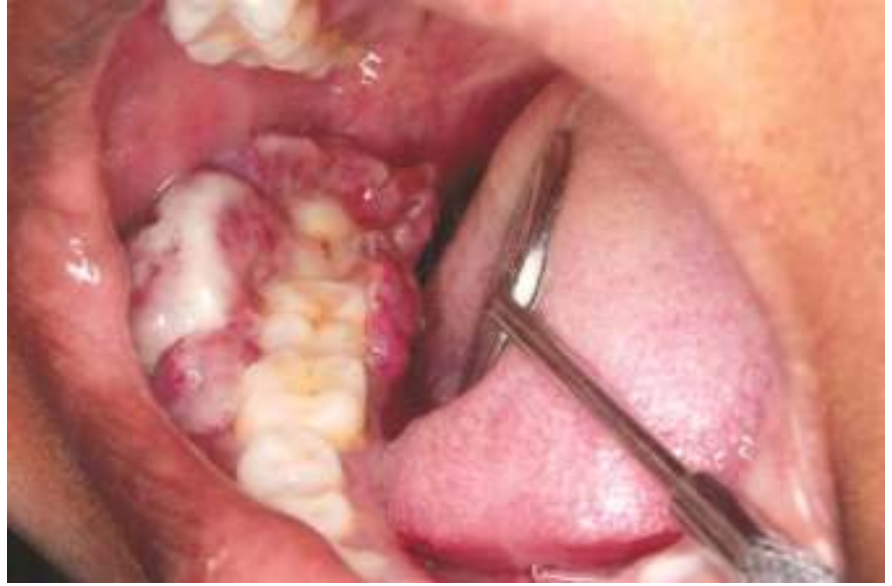

Fig 1: Pre operative view of the lesion

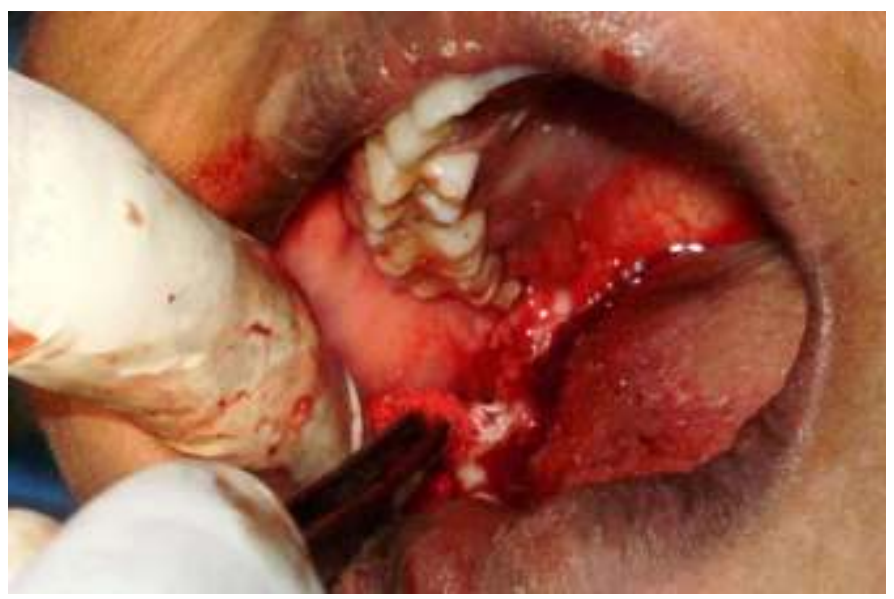

Fig 3: After the excision

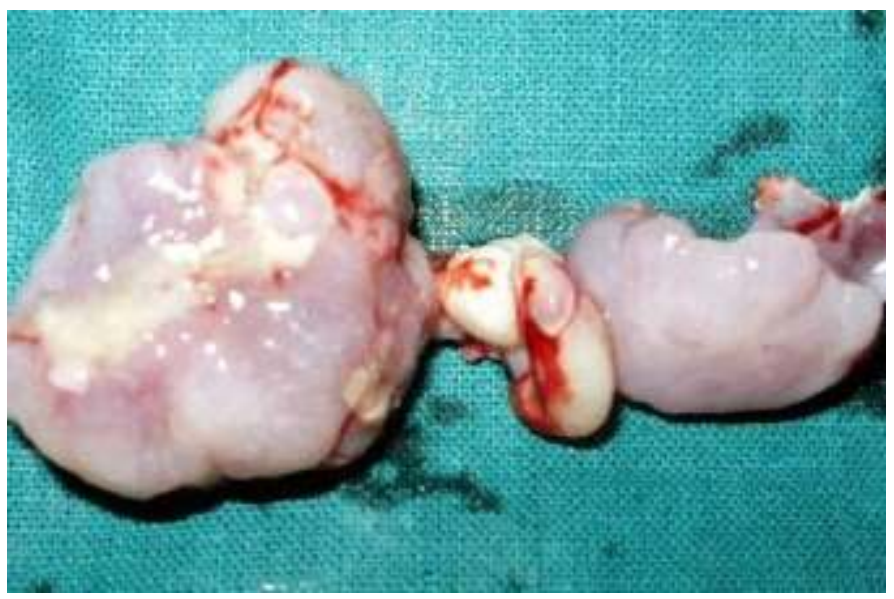

Fig 5: Excised tissue

size and gradually increasing in size. On extra oral examination no facial asymmetry was noticed. On intra oral examination swelling of measuring about $1.5 \mathrm{cmx} 2 \mathrm{cms}$ size is present at 46 and 47 (fig 7) with a pedunculated base. On palpation swelling had a smooth texture which is firm in consistency and bleeds on probing. Lesion was painless and asymptomatic. There were no signs of local etiological factors pertaining to the lesion site. No mobility of teeth in relation to the lesion. Lesion was excised by using B.P blade no. 12 from its base (fig 8) and the tissue was sent for histopatholgical examination.

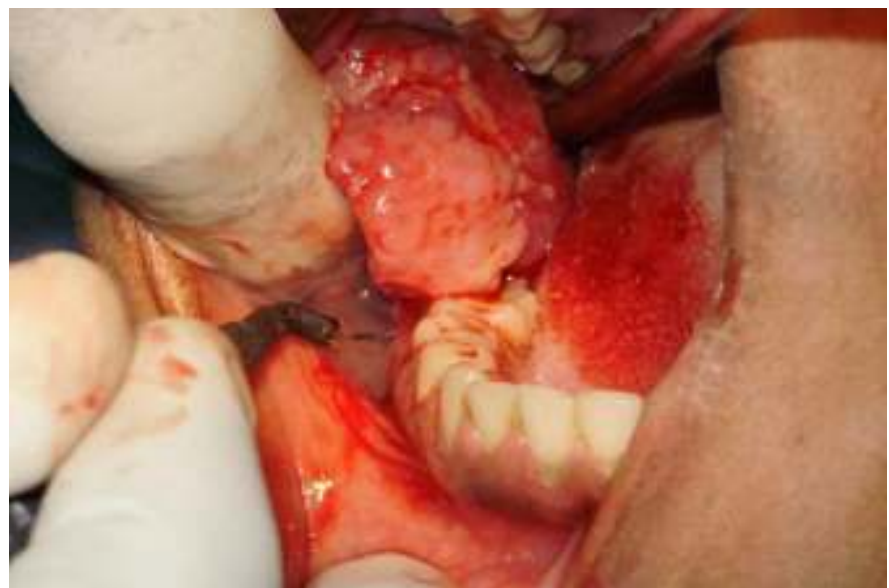

Fig 2: Excision with electrocautery

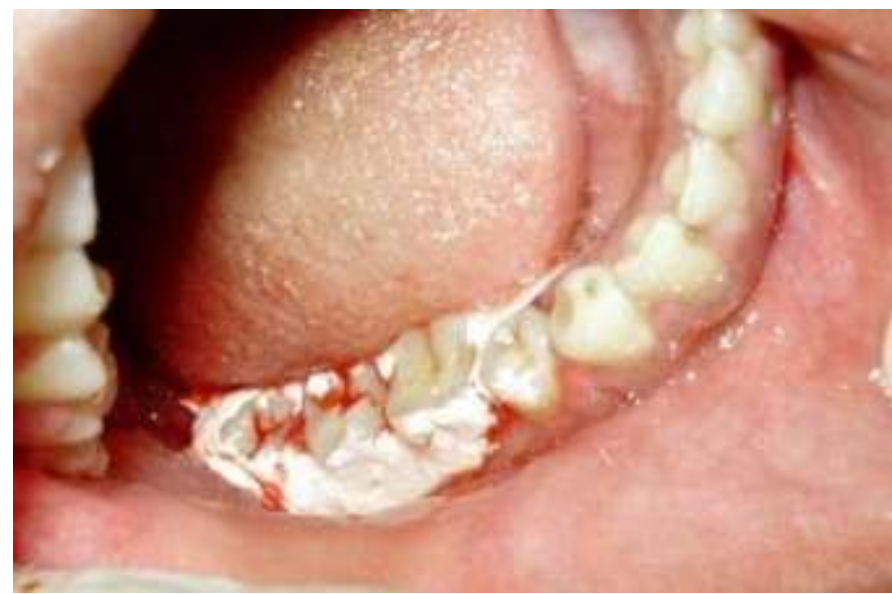

Fig 4: Periodontal pack after the excision

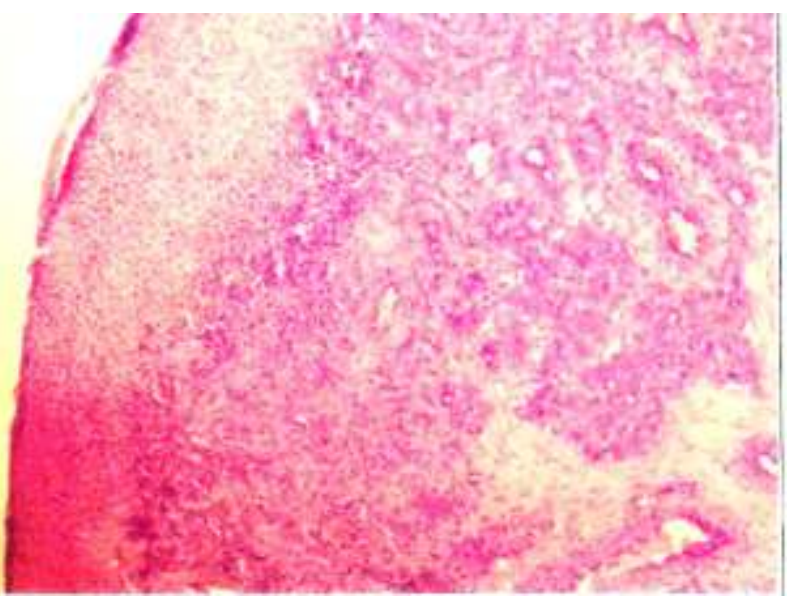

Fig 6: Histopathologysection (10X) showing connective tissue stroma with numerous blood vessels of variable proliferating fibroblasts and dense inflammatory cell infiltration and pyogenic membrane formation.

\section{DISCUSSION}

Pyogenic granuloma is a hyperplastic reaction of a connective tissue to inflammation. It was first reported in the English literature by hullehen [7] and first described in man by two French surgeons, Poncet and Dor in 1897, named it as botryomycosis hominis [8]. Even though, it is not a true granuloma in actuality, it is a capillary hemangioma of lobular 


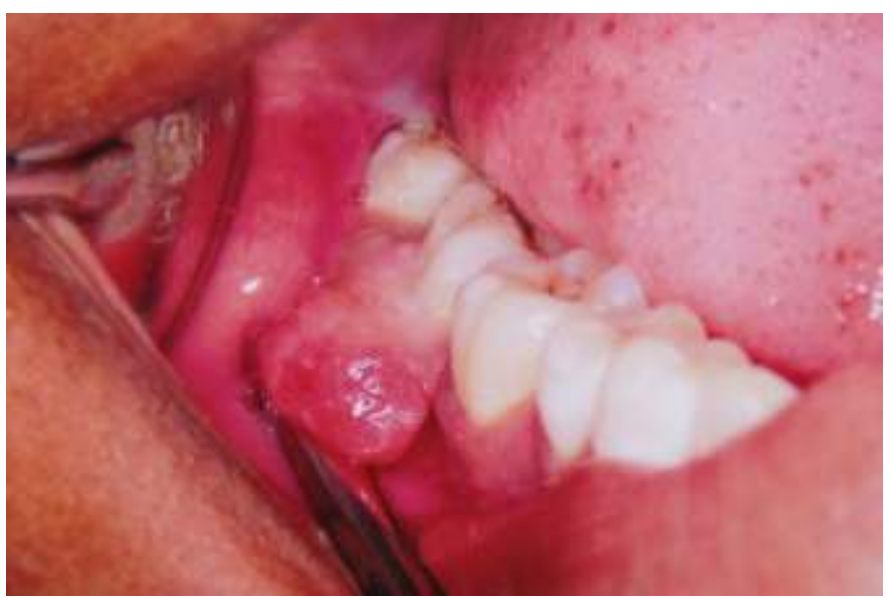

Fig 7:Pre operative view of the lesion

subtype, which is quite often prone to bleeding[9]. It is not truly pyogenic as it does not produce pus. Term Pyogenic granuloma or granuloma pyogenicum was introduced by Hartzell [10]. Angelopoulos AP proposed the term "haemangiomatous granuloma" as it accurately resembles the histopathologic picture 'haemangioma like' and the 'inflammatory like'[11]. Term "Granuloma Telangiectacticum" was given by Cawson et al.[3] Till date two types of pyogenic granuloma are noted, one with lobular capillary haemangioma and non- lobular capillary haemangionma[12]. The incidence of pyogenic granuloma has been described as between 26.8 and $32 \%$ of all reactive lesions.[13]

Several investigators suggest that response to various causative/ local irritative factors and poor oral hygiene within the gingival crevice leads to extensive proliferation of connective tissue[14].Majority of pyogenic granulomas are found on marginal gingival [15] and occurs during second decades of life.[16] These appear as smooth, lobulated appearance with a size ranging from several millimeters to centimeters with significant bone loss if is involved with alveolar bone. Murata et al shown that cytokine bFGF immune-localization in gingiva at various stages of pyogenic granulomas are suggestive of maximum numbers of cytokines are released from macrophages and mast cells thus aiding in neo-vascularization of the tissue.[17]

Most theories on pathogenesis revolve around PG as a hyperplastic, neovascular response to an angiogenic stimulus with imbalance of promoters and inhibitors [18,19]. Angiogenic growth factors such as vascular endothelial growth factor (VEGF) and decorin, transcription factors (pATF2 and pSTAT3), and signal transduction pathways (MAPK) are overexpressed in PGs, but their exact role is undetermined[19,20,21].

Yung et al suggested hormonal influence on the basis of the observation that pregnancy tumor that occurs in the pregnant women also arises from the gingiva and has the same microscopic appearance [5]. Hosseini et al. stated that there are clinical observations that gingiva may be enlarged during pregnancy and may atrophy during menopause and stated that gingiva may become "Target Organ" by certain hormones like estrogen and progesterone due to which, there is gingival overgrowth in pregnancy and atrophy in menopause.[22] Though, these two hormones markedly rise in pregnancy, these do not exert a direct effect on gingiva but, they may influence the endothelium of pyogenic granulomas.[23] Few studies reported that, in pregnancy, inflammatory cells and fibroblasts are inhibited and the progesterone regulates the metabolism of both. Co-existence

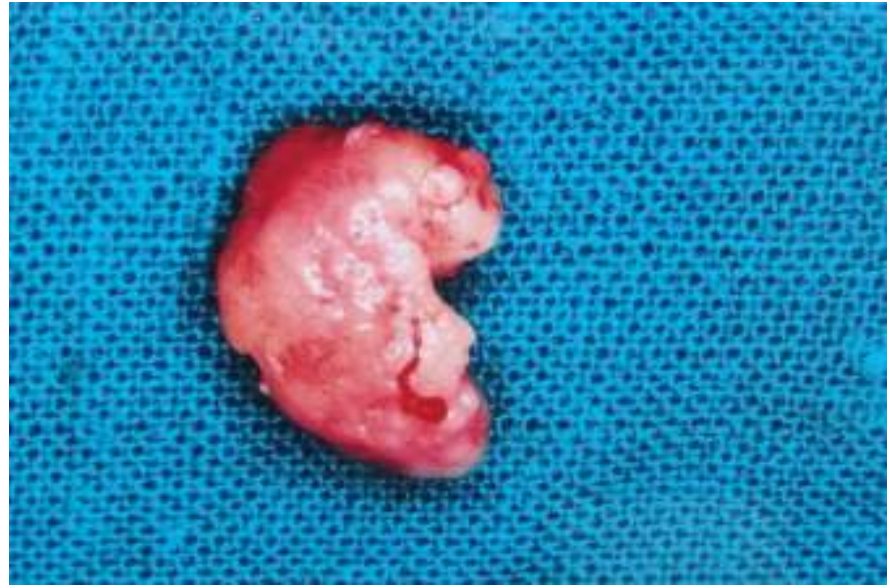

\section{Fig 8: Excised tissue}

of these factors may cause tissue reaction to local factors resulting in inflammation. Despite of all these, some authors have stated that this lesion appears equally in both males and females, and disagreed with hormonal influence during pregnancy.[24] However, its etio-pathogenesis is still debatable. Despite of having different and various surgical techniques, apart from surgical excision, like cryosurgery, lasers (ND:YAG) and sclerotherapy by using injection of corticosteroid and sodium tetradecyl sulfate, surgical excision with a safety distance of $1 \mathrm{~mm}$ is the treatment of choice is a successful treatment of choice and patients should be advised about the importance of oral hygiene maintenance. Further studies are required to conclude its relation with/during pregnancy.

\section{CONCLUSION}

Pyogenic granuloma is a non neoplastic inflammatory enlargement commonly seen in the oral cavity. Although, predeliction of this lesion is more during pregnancy, causative factors are unclear. Proper diagnosis and treatment plan with effective education and motivation would prevent its recurrence.

\section{REFERENCES}

1. Reet Kamal, Parveen Dahiya1, Abhiney Puri. Oral pyogenic granuloma: Various concepts of etiopathogenesis. J Oral Maxillofac Pathol. 2012;16:7982.

2. Layfield LL, Shopper TP, Weir JC. A diagnostic survey of biopsied gingival lesions. J Dent Hyg 1995;69:175-79.

3. B.W.Neville, D.D.Damn, C.M. Allen, and J.E.Bouquot, Oral and Maxillofacial Pathology, WB Saunders, Philadelphia, Pa, USA, 2nd edition, 2002.

4. Jeevanand Deshmukh, Vinaya Kumar Kulkarni, Girish Katti, Sachin Deshpande. Pyogenic Granuloma - An Unusual Presentation In Pediatric Patient - A Case Report. Indian Journal of Dental Sciences.2013;5 (1);90-93.

5. Cawson RA, Binnie WH, Speight PM, Barrett AW, Wright JM. Lucas Pathology of tumors of oral tissues. 5th ed. Missouri: Mosby; 1998. pp. 252-254.

6. Sumanth Shivaswamy, Nazia Siddiqui, A. Sanjay Jain, Ajit Koshy, Sonal Tambwekar, Akhil Shankar. A rare case of generalized pyogenic granuloma: A case report. Quintessence Int. 2011; 42: 493-99.

7. Hullihen SP Case of aneurism by anastomosis of the 
superior maxilla. Am J Dent Sc 1844; 4: 160-62.

8. Ferry AP, Zimmerman LE. Granuloma pyogenicum of limbus. Arch Ophthalmol.1965; 74: 229-230.

9. JafarzadehH, Sanatkhani M, MohtashamN. Oral pyogenic granuloma: a review" J Oral Sci.2006; 48 (4): $167-75$.

10. Hartzell MB, granuloma pyogenicum J Cutan Dis Syph.1904; 22.520-25.

11. Angelopoulos AP .pyogenic granuloma of the oral cavity: Stastical analysis of its clinical features. J Oral Surg. 1971;29: 840-7.

12. Epivatinos A, Antoniades D, Zaraboukas T, Zairi E, PoulopoulosA, Kiziridou A, IordanidisS. Pyogenic granuloma of the oral cavity: Comparitive study of clinicpathological and immuno-histological features. Pathol Int.2005; 55,391-97.

13. Selvamuthukumar, Nalini Ashwath, Anand. Unusual Presentation of Pyogenic Granuloma of Buccal Mucosa. Journal of Indian Academy of Oral Medicine and Radiology, 2010; 22 (4):S45-47.

14. Regezi JA, Sciubba JJ, Jordan RC, Oral Pathology: Clinical pathological considerations. Philadelphia. WB Saunders; 2003; 4thed: 115-6.

15. Vilmann A, Vilmann P, Vilmann H. Pyogenic granuloma: evaluation of oral conditions. Br J Oral Maxillo fac Surg 1986; 24(5): 376-82.

16. Zain R, Khoo S, Yeo J. Oral pyogenic granuloma clinical analysis of 304 cases. Singapore Dent J 1995; 20(1): 8-10.

17. Murata M, Hara K, Saku T, Dynamic distribution of basic fibroblast growth factors during epulis formation: An immuno-histochemical study in an enhanced healing process of the gingiva. J Oral Pathol Med: 1997; 26: 22432.

18. Giblin AV, Clover AJ, Athanassopoulos A, Budny PG. Pyogenic granuloma - the quest for optimum treatment: audit of treatment of 408 cases. J Plast Reconstr AesthetSurg 2007; 60:1030.

19. Piguet V, Borradori L. Pyogenic granuloma-like lesions during capecitabine therapy. Br J Dermatol 2002; 147:1270.

20. Chen SY, Takeuchi S, Urabe K, et al. Over expression of phosphorylated-ATF2 and STAT3 in cutaneous angiosarcoma and pyogenic granuloma. J Cutan Pathol $2008 ; 35: 722$.

21. Arbiser JL, Weiss SW, Arbiser ZK, et al. Differential expression of active mitogen-activated protein kinase in cutaneous endothelial neoplasms: implications for biologic behavior and response to therapy. J Am Acad Dermatol 2001; 44:193.

22. Hosseini FH, Tirgari $F$ and Shaigan S. Immunohistochemical analysis of estrogen and progesterone receptor expression in gingival lesions. Iran J Public Health.2006;35:38-41.

23. Whittaker SB, Bouquot JE, Alimario AE, Whittaker TJ Jr, Identification and semi quantification of oestrogen and progesterone receptors in pyogenic granulomas of pregnancy, Oral Surg, Oral Med, Oral Pathol.1994:78:75560.

24. Ojanotak-Hari AO, Harri MP, Hurttia HM, Sewon LA. Tissue metabolism of progesterone in pregnancy gingivitis and granulomas. J Clin Periodontol.1991;18:262-6 